\title{
1,3-Dibromo-5,5-dimethylhydantoin as promoter for glycosylations using thioglycosides
}

\author{
Fei-Fei $\mathrm{Xu}^{1,2}$, Claney L. Pereira*1,3 and Peter H. Seeberger ${ }^{* 1,2}$
}

\author{
Full Research Paper \\ Address: \\ ${ }^{1}$ Department of Biomolecular Systems, Max Planck Institute of \\ Colloids and Interfaces, Am Mühlenberg 1, 14476 Potsdam, \\ Germany, ${ }^{2}$ Department of Chemistry and Biochemistry, Freie \\ Universität Berlin, Arnimallee 22, 14195 Berlin, Germany and \\ ${ }^{3}$ Vaxxilon Deutschland $\mathrm{GmbH}$, Magnusstraße 11, 12489 Berlin, \\ Germany \\ Email: \\ Claney L. Pereira* - claney.pereira@vaxxilon.com; \\ Peter H. Seeberger ${ }^{*}$ - peter.seeberger@mpikg.mpg.de \\ * Corresponding author \\ Keywords: \\ automated glycan assembly; 1,3-dibromo-5,5-dimethylhydantoin; \\ glycosylation; promoter; thioglycosides \\ Beilstein J. Org. Chem. 2017, 13, 1994-1998. \\ doi:10.3762/bjoc.13.195 \\ Received: 20 July 2017 \\ Accepted: 07 September 2017 \\ Published: 22 September 2017 \\ Associate Editor: B. Stoltz \\ (C) 2017 Xu et al.; licensee Beilstein-Institut. \\ License and terms: see end of document.
}

\begin{abstract}
1,3-Dibromo-5,5-dimethylhydantoin (DBDMH), an inexpensive, non-toxic and stable reagent, is a competent activator of thioglycosides for glycosidic bond formation. Excellent yields were obtained when triflic acid (TfOH) or trimethylsilyl trifluoromethanesulfonate (TMSOTf) were employed as co-promoters in solution or automated glycan assembly on solid phase.
\end{abstract}

\section{Introduction}

Thioglycosides are versatile glycosylating agents that are commonly used in oligosaccharide synthesis due to their accessibility, stability, compatibility with various reaction conditions, and orthogonality to other donors [1-5]. Different electrophilic/ thiophilic reagents have been developed as promoters to activate thioglycoside donors [3,6-18]. However, most of those activators are expensive and toxic [5,17,19]. Poor solubility complicates the use of some promoters during automated glycan assembly [20-23], while the instability of some activators in solution requires them to be freshly prepared prior to use [2426]. Here, we describe a promoter system based on the commer- cially available, inexpensive 1,3-dibromo-5,5-dimethylhydantoin (DBDMH) for the activation of thioglycosides.

DBDMH, a white to pale-brown powder that is readily soluble in most organic solvents, including dichloromethane, is sold under the trade name Brom-55 and used as swimming pool sanitizer, as industrial brominating agent for ethylene propylene diene monomer rubber to improve ozone resistance, as additive in plastics to promote photodegradation and as a fungicide to preserve fresh fruits [27]. In synthetic chemistry, DBDMH acts as a thiophilic activator in the conversion of 
dithioacetals to the corresponding $O, O$-acetals [28-30], as well as in the synthesis of heparin mimetics [31]. We considered DBDMH as a readily available alternative promoter for glycosylations involving thioglycosides.

\section{Results and Discussion}

Initially, the capability of DBDMH to activate thioglycoside $\mathbf{1}$ [32] in order to glycosylate the primary hydroxy group present in D-glucose acceptor 2 [33] was explored without any additives (Table 1, entry 1). This initial experiment furnished disaccharide 3 , albeit in modest yield (43\%). When $\mathrm{TfOH}$ or TMSOTf ( $10 \mathrm{~mol} \%)$ were added as co-promoter, the yield increased to more than $90 \%$ (Table 1, entries 2 and 3). Next, the amount of the reagent required for activation was studied (Table 1, entries 3-5). Substoichiometric amounts of DBDMH ( 0.7 equiv) in the presence of co-promoter suffice to produce the disaccharide efficiently. The DBDMH/TfOH activation system is temperature insensitive as it furnishes the product from $-78^{\circ} \mathrm{C}$ to room temperature, although most disaccharide 3 is formed at $-40{ }^{\circ} \mathrm{C}$ (Table 1, entries 3 and 6-9).

Next, the scope of the new activation system was investigated by using a variety of glycosyl donors 4-10 [34-38] containing C-2 participating groups to ensure 1,2-trans-glycoside formation (Table 2). Each glycosylating agent was reacted with D-glucose acceptors 2 (Table 2, entries 1-8) and 11 [39]
(Table 2, entries 9-16) with a free hydroxy group at C-6 and C-4 position, respectively. The DBDMH/TfOH system activates glycosyl donors including neutral monosaccharides of different configurations (D-gluco 5 and 6, D-galacto 1 and 4, D-manno 8, L-rhamno 9), amino sugar 7 and uronic acid 10. All thioglycosides reacted equally well, irrespective of their aglycons (SEt or STol). This promoter is compatible with most commonly used protecting groups, except some electron-rich groups like 4-methoxybenzyl ethers that may be partly brominated under these conditions [40].

To probe the scope of DBDMH/TfOH-mediated 1,2-cis-glycosylation, perbenzylated galactosyl donor 12 [41] (Table 3, entries 1-4) and galactosyl donor 13 [42] (Table 3, entries 5 and 6) as well as glucosyl donor $\mathbf{1 4}$ (Table 3 , entries 7 and 8) were reacted with acceptor $\mathbf{2}$ in the presence of DBDMH. Electronrich ('armed') thioglycosides [43] are more readily activated as the reaction of perbenzylated donor $\mathbf{1 2}$ in dichloromethane at $-78^{\circ} \mathrm{C}$ afforded the disaccharide with excellent yield but low stereoselectivity. The $\alpha / \beta$ ratio, determined by supercritical fluid chromatography (SFC), shifted significantly toward the $\alpha$-isomer with ether [44] and toward the $\beta$-isomer when acetonitrile [45] was used as co-solvent. With all these donors, the $\alpha$-stereoselectivity increased at higher temperature [46]. Donor 13, containing a remote participating group, produced the disaccharide with better $\alpha$-selectivity $[22,42]$.

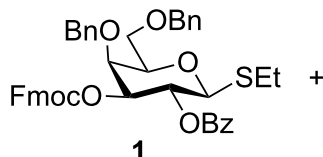

1

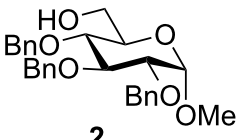<smiles>CC1(C)C(=O)N(Br)C(=O)N1Br</smiles>

DBDMH

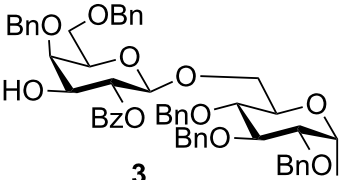

3

\begin{tabular}{|c|c|c|c|c|}
\hline Entry $^{a}$ & DBDMH (equiv ${ }^{b}$ ) & Co-promoter $(10 \mathrm{~mol} \% \mathrm{~b})$ & $T\left({ }^{\circ} \mathrm{C}\right)$ & Yield $^{\mathrm{C}}(\%)$ \\
\hline 1 & 0.7 & - & -40 & 43 \\
\hline 2 & 0.7 & TMSOTf & -40 & 93 \\
\hline 3 & 0.7 & $\mathrm{TfOH}$ & -40 & 92 \\
\hline 4 & 0.5 & $\mathrm{TfOH}$ & -40 & 85 \\
\hline 5 & 1.0 & $\mathrm{TfOH}$ & -40 & 94 \\
\hline 6 & 0.7 & $\mathrm{TfOH}$ & -78 & 83 \\
\hline 7 & 0.7 & $\mathrm{TfOH}$ & -20 & 87 \\
\hline 8 & 0.7 & $\mathrm{TfOH}$ & 0 & 88 \\
\hline 9 & 0.7 & $\mathrm{TfOH}$ & $\mathrm{rt}$ & 79 \\
\hline
\end{tabular}

aReaction conditions: donor $(51 \mu \mathrm{mol})$, acceptor $(43 \mu \mathrm{mol})$, dichloromethane; quenched with triethylamine. Fmoc protecting group was removed during the quenching process in the presence of triethylamine. ${ }^{b}$ Equivalents calculated relative to the amount of donor. ${ }^{c}$ Only isolated yields are reported. 
Table 2: 1,2-Trans-glycosylation activated by DBDMH with a variety of building blocks.

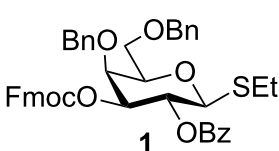

$1 \mathrm{OBz}$

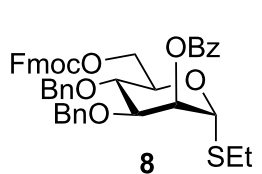

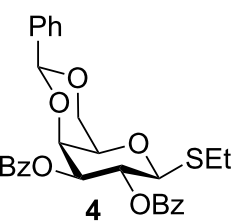
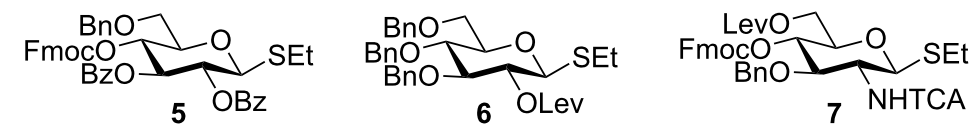
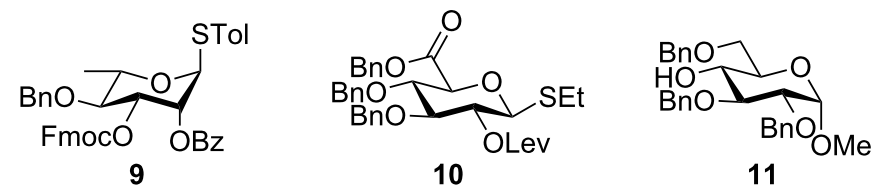

\begin{tabular}{|c|c|c|c|c|c|c|c|}
\hline Entry $^{a}$ & Donor & Acceptor & Yield ${ }^{b}(\%)$ & Entrya & Donor & Acceptor & Yield ${ }^{b}(\%)$ \\
\hline 1 & 1 & 2 & 92 & 9 & 1 & 11 & 88 \\
\hline 2 & 4 & 2 & 95 & 10 & 4 & 11 & 88 \\
\hline 3 & 5 & 2 & 98 & 11 & 5 & 11 & 87 \\
\hline 4 & 6 & 2 & 94 & 12 & 6 & 11 & 89 \\
\hline 5 & 7 & 2 & 91 & 13 & 7 & 11 & 60 \\
\hline 6 & 8 & 2 & 96 & 14 & 8 & 11 & 89 \\
\hline 7 & 9 & 2 & 91 & 15 & 9 & 11 & 86 \\
\hline 8 & 10 & 2 & 39 & 16 & 10 & 11 & 45 \\
\hline
\end{tabular}

aAll reactions were carried out at $-40^{\circ} \mathrm{C}$ in dichloromethane with 0.7 equiv DBDMH and $10 \mathrm{~mol} \%$ TfOH as promoter. ${ }^{b}$ Only isolated yields are reported.

Table 3: 1,2-Cis-glycosylation activated by DBDMH.

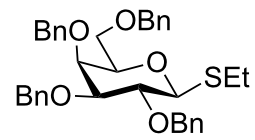

12

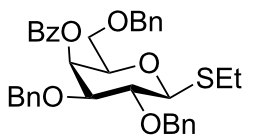

13

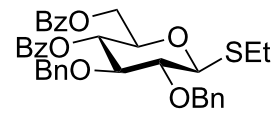

14

\begin{tabular}{|c|c|c|c|c|c|c|}
\hline Entrya & Donor & Acceptor & Solvent & $T\left({ }^{\circ} \mathrm{C}\right)$ & Yield ${ }^{b}(\%)$ & $\alpha / \beta$ ratio $^{c}$ \\
\hline 1 & 12 & 2 & $\mathrm{DCM} / \mathrm{Et}_{2} \mathrm{O}^{\mathrm{d}}$ & -78 & 94 & $1: 1.4$ \\
\hline 2 & 12 & 2 & $\mathrm{DCM}$ & -78 & 94 & $1: 2.7$ \\
\hline 3 & 12 & 2 & $\mathrm{DCM} / \mathrm{MeCN}^{\mathrm{d}}$ & -78 & 93 & $1: 11.7$ \\
\hline 4 & 12 & 2 & $\mathrm{DCM}$ & -40 & 67 & $1: 1.3$ \\
\hline 5 & 13 & 2 & DCM & -78 & 72 & $4.6: 1$ \\
\hline 6 & 13 & 2 & $\mathrm{DCM}$ & -40 & 50 & 11.8:1 \\
\hline 7 & 14 & 2 & DCM & -78 & 76 & $1: 1.1$ \\
\hline 8 & 14 & 2 & DCM & -40 & 69 & $1: 1$ \\
\hline
\end{tabular}

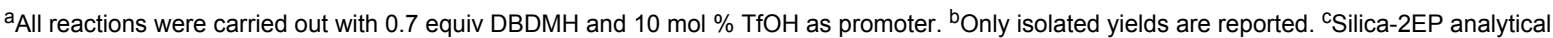
column was used to determine the $\alpha / \beta$ ratio when using SFC. Isopropanol was used as co-solvent for the mobile phase. ${ }^{d}$ The ratio of solvents is $2: 1(v / v)$.

Automated glycan assembly is the most rapid means to access complex oligosaccharides [20,47]. Ideally, stable and non-toxic reagents should be used on such instruments. The automated synthesis of disaccharide $\mathbf{1 6}$ served to assess the suitability of the DBDMH/TMSOTf activation system using functionalized resin 15 [48] as solid support (Scheme 1). After two coupling cycles with building block $\mathbf{8}$ followed by UV-cleavage, disaccharide $\mathbf{1 6}$ was obtained in $63 \%$ isolated yield. 


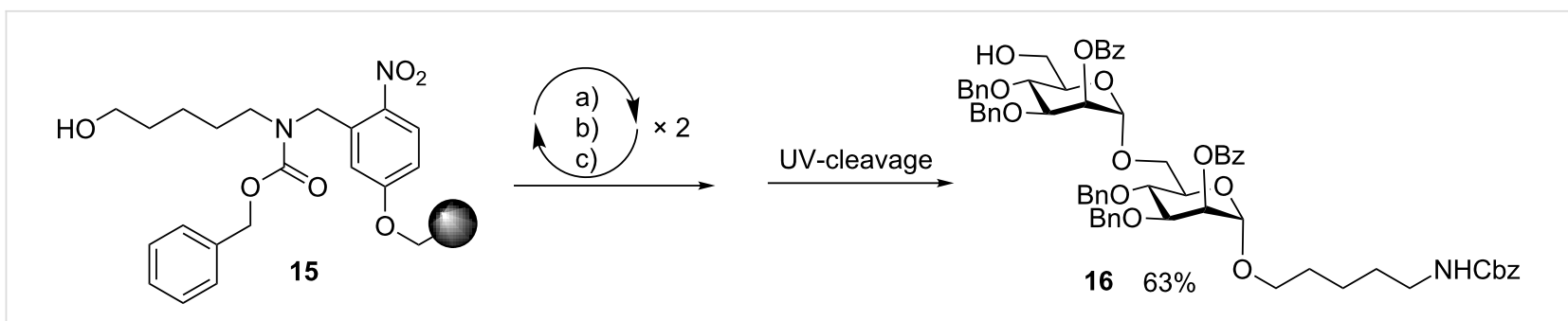

Scheme 1: DBDMH as promotor for automated glycan assembly. Modules: a) acidic wash; b) glycosylation using DBDMH/TMSOTf, 8; c) Fmoc deprotection.

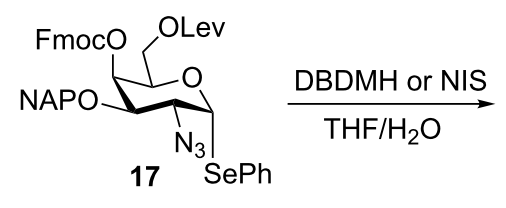

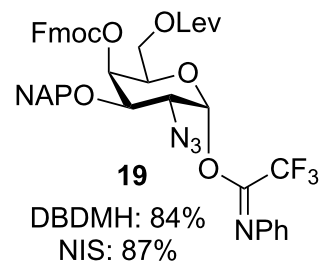

Scheme 2: Hydrolysis of glycosyl selenide 17 with $\mathrm{DBDMH}$.

Moreover, DBDMH performs as well as $N$-iodosuccinimide (NIS) in activating phenyl selenoglycoside $\mathbf{1 7}$ in the presence of water to furnish hemiacetal $\mathbf{1 8}$ en route to glycosyl imidate $\mathbf{1 9}$ (Scheme 2).

\section{Conclusion}

The inexpensive reagent DBDMH has been demonstrated to be a powerful promoter for the activation of thioglycosides. This promoter is readily available, highly soluble, and shelf-stable. A variety of substrates containing diverse protecting groups have been investigated with promising results, while the stereoselectivity of the reactions follows reported trends. This promoter system was successfully used for automated glycan assembly.

\section{Supporting Information}

\section{Supporting Information File 1}

Experimental details and full characterization data of all new compounds.

[http://www.beilstein-journals.org/bjoc/content/ supplementary/1860-5397-13-195-S1.pdf]

\section{Acknowledgements}

We gratefully acknowledge the Max Planck Society for financial support. We thank Dr. Martina Delbianco for help with automated glycan assembly, Ms. Priya Bharate for providing building block 8, Dr. Madhu Emmadi for building block 17, Dr. Lennart Lykke for building block 9 and Ms. Eva Settels for assistance with performing SFC and HPLC analysis.

\section{ORCID ${ }^{\circledR}$ iDs}

Claney L. Pereira - https://orcid.org/0000-0003-1972-7907

\section{References}

1. Codée, J. D. C.; Litjens, R. E. J. N.; van den Bos, L. J.; Overkleeft, H. S.; van der Marel, G. A. Chem. Soc. Rev. 2005, 34, 769-782. doi:10.1039/B417138C

2. Zhang, Z.; Ollmann, I. R.; Ye, X.-S.; Wischnat, R.; Baasov, T.; Wong, C.-H. J. Am. Chem. Soc. 1999, 121, 734-753. doi:10.1021/ja982232s

3. Lian, G.; Zhang, X.; Yu, B. Carbohydr. Res. 2015, 403, 13-22. doi:10.1016/j.carres.2014.06.009

4. Frihed, T. G.; Pedersen, C. M.; Bols, M. Angew. Chem., Int. Ed. 2014, 53, 13889-13893. doi:10.1002/anie.201408209

5. Ferrier, R. J.; Hay, R. W.; Vethaviyasar, N. Carbohydr. Res. 1973, 27, 55-61. doi:10.1016/S0008-6215(00)82424-6

6. Zhu, X.; Schmidt, R. R. Angew. Chem., Int. Ed. 2009, 48, 1900-1934. doi:10.1002/anie.200802036

7. Veeneman, G. H.; van Leeuwen, S. H.; van Boom, J. H. Tetrahedron Lett. 1990, 31, 1331-1334. doi:10.1016/S0040-4039(00)88799-7

8. Nicolaou, K. C.; Seitz, S. P.; Papahatjis, D. P. J. Am. Chem. Soc. 1983, 105, 2430-2434. doi:10.1021/ja00346a053

9. Petitou, M.; Duchaussoy, P.; Lederman, I.; Choay, J.; Sinaÿ, P.; Jacquinet, J.-C.; Torri, G. Carbohydr. Res. 1986, 147, 221-236. doi:10.1016/S0008-6215(00)90633-5

10. Ercegovic, T.; Meijer, A.; Magnusson, G.; Ellervik, U. Org. Lett. 2001, 3, 913-915. doi:10.1021/ol015547c

11. Crich, D.; Smith, M. J. Am. Chem. Soc. 2001, 123, 9015-9020. doi:10.1021/ja0111481

12. Durón, S. G.; Polat, T.; Wong, C.-H. Org. Lett. 2004, 6, 839-841. doi:10.1021/ol0400084

13. Tatai, J.; Fügedi, P. Org. Lett. 2007, 9, 4647-4650. doi:10.1021/ol702139u 
14. Nokami, T.; Shibuya, A.; Tsuyama, H.; Suga, S.; Bowers, A. A.; Crich, D.; Yoshida, J.-i. J. Am. Chem. Soc. 2007, 129, 10922-10928. doi:10.1021/ja072440x

15. Wever, W. J.; Cinelli, M. A.; Bowers, A. A. Org. Lett. 2013, 15, 30-33. doi:10.1021/ol302941q

16. Goswami, M.; Ellern, A.; Pohl, N. L. B. Angew. Chem., Int. Ed. 2013, 52, 8441-8445. doi:10.1002/anie.201304099

17. Vibhute, A. M.; Dhaka, A.; Athiyarath, V.; Sureshan, K. M. Chem. Sci. 2016, 7, 4259-4263. doi:10.1039/C6SC00633G

18. Basu, N.; Maity, S. K.; Chaudhury, A.; Ghosh, R. Carbohydr. Res. 2013, 369, 10-13. doi:10.1016/j.carres.2013.01.001

19. Lear, M. J.; Yoshimura, F.; Hirama, M. Angew. Chem., Int. Ed. 2001, 40, 946-949. doi:10.1002/1521-3773(20010302)40:5<946::AID-ANIE946>3.0.CO;2G

20. Plante, O. J.; Palmacci, E. R.; Seeberger, P. H. Science 2001, 291, 1523-1527. doi:10.1126/science.1057324

21. Lai, C.-H.; Hahm, H. S.; Liang, C.-F.; Seeberger, P. H. Beilstein J. Org. Chem. 2015, 11, 617-621. doi:10.3762/bjoc.11.69

22. Hahm, H. S.; Hurevich, M.; Seeberger, P. H. Nat. Commun. 2016, 7, No. 12482. doi:10.1038/ncomms 12482

23. Fair, R. J.; Hahm, H. S.; Seeberger, P. H. Chem. Commun. 2015, 51, 6183-6185. doi:10.1039/C5CC01368B

24. Dasgupta, F.; Garegg, P. J. Carbohydr. Res. 1988, 177, c13-c17. doi:10.1016/0008-6215(88)85071-7

25. Martichonok, V.; Whitesides, G. M. J. Org. Chem. 1996, 61, 1702-1706. doi:10.1021/jo951711w

26. Huang, X.; Huang, L.; Wang, H.; Ye, X.-S. Angew. Chem., Int. Ed. 2004, 43, 5221-5224. doi:10.1002/anie.200460176

27. Kolvari, E.; Ghorbani-Choghamarani, A.; Salehi, P.; Shirini, F.; Zolfigol, M. A. J. Iran. Chem. Soc. 2007, 4, 126-174. doi:10.1007/BF03245963

28. Madhusudan, S. K.; Misra, A. K. Carbohydr. Res. 2005, 340, 497-502. doi:10.1016/j.carres.2004.12.002

29. Madhusudan, S. K.; Misra, A. K. Eur. J. Org. Chem. 2005, 3196-3205. doi:10.1002/ejoc.200500081

30. Bai, Y.; Lowary, T. L. J. Org. Chem. 2006, 71, 9658-9671. doi:10.1021/jo061713o

31. El Hadri, A.; Petitou, M. Synthetic pentasaccharides having short half-life and high activity. WO Patent WO2012172104 A1, Dec 20, 2012.

32. Hahm, H. S.; Liang, C.-F.; Lai, C.-H.; Fair, R. J.; Schuhmacher, F.; Seeberger, P. H. J. Org. Chem. 2016, 81, 5866-5877. doi:10.1021/acs.joc.6b00554

33. Viuff, A. H.; Besenbacher, L. M.; Kamori, A.; Jensen, M. T.; Kilian, M.; Kato, A.; Jensen, H. H. Org. Biomol. Chem. 2015, 13, 9637-9658. doi:10.1039/C5OB01281C

34. Lindberg, J.; Svensson, S. C. T.; Påhlsson, P.; Konradsson, P. Tetrahedron 2002, 58, 5109-5117. doi:10.1016/S0040-4020(02)00473-8

35. Nokami, T.; Tsuyama, H.; Shibuya, A.; Nakatsutsumi, T.; Yoshida, J.-i. Chem. Lett. 2008, 37, 942-943. doi:10.1246/cl.2008.942

36. Watt, G. M.; Boons, G.-J. Carbohydr. Res. 2004, 339, 181-193. doi:10.1016/j.carres.2003.10.029

37. Hahm, H. S.; Broecker, F.; Kawasaki, F.; Mietzsch, M.; Heilbronn, R.; Fukuda, M.; Seeberger, P. H. Chem 2017, 2, 114-124. doi:10.1016/j.chempr.2016.12.004

38. Lisboa, M. P.; Khan, N.; Martin, C. E.; Xu, F.-F.; Reppe, K.; Geissner, A.; Govindan, S.; Witzenrath, M.; Pereira, C. L.; Seeberger, P. H. Proc. Natl. Acad. Sci. U. S. A. 2017, accepted.
39. DeNinno, M. P.; Etienne, J. B.; Duplantier, K. C. Tetrahedron Lett. 1995, 36, 669-672. doi:10.1016/0040-4039(94)02348-F

40. Chassaing, C.; Haudrechy, A.; Langlois, Y. Tetrahedron Lett. 1997, 38, 4415-4416. doi:10.1016/S0040-4039(97)00943-X

41. Ekholm, F. S.; Ardá, A.; Eklund, P.; André, S.; Gabius, H.-J.; Jiménez-Barbero, J.; Leino, R. Chem. - Eur. J. 2012, 18, 14392-14405. doi:10.1002/chem.201200510

42. Demchenko, A. V.; Rousson, E.; Boons, G.-J. Tetrahedron Lett. 1999, 40, 6523-6526. doi:10.1016/S0040-4039(99)01203-4

43. Veeneman, G. H.; van Boom, J. H. Tetrahedron Lett. 1990, 31, 275-278. doi:10.1016/S0040-4039(00)94391-0

44. Demchenko, A.; Stauch, T.; Boons, G.-J. Synlett 1997, 818-820. doi:10.1055/s-1997-5762

45. Braccini, I.; Derouet, C.; Esnault, J.; de Penhoat, C. H.; Mallet, J.-M.; Michon, V.; Sinaÿ, P. Carbohydr. Res. 1993, 246, 23-41. doi:10.1016/0008-6215(93)84021-W

46. Kalikanda, J.; Li, Z. J. Org. Chem. 2011, 76, 5207-5218. doi:10.1021/jo1025157

47. Seeberger, P. H. Acc. Chem. Res. 2015, 48, 1450-1463. doi:10.1021/ar5004362

48. Eller, S.; Collot, M.; Yin, J.; Hahm, H. S.; Seeberger, P. H. Angew. Chem., Int. Ed. 2013, 52, 5858-5861. doi:10.1002/anie.201210132

\section{License and Terms}

This is an Open Access article under the terms of the Creative Commons Attribution License (http://creativecommons.org/licenses/by/4.0), which permits unrestricted use, distribution, and reproduction in any medium, provided the original work is properly cited.

The license is subject to the Beilstein Journal of Organic Chemistry terms and conditions:

(http://www.beilstein-journals.org/bjoc)

The definitive version of this article is the electronic one which can be found at: $\underline{\text { doi:10.3762/bjoc. } 13.195}$ 